\title{
Crystallography and Magnetism of Two 1-(4'-Nitroxylphenyl)pyrroles.
}

\author{
Zeynep Delen, Paul M. Lahti* \\ Department of Chemistry, University of Massachusetts, Amherst, MA 01003 \\ lahti@chem.umass.edu
}

Item

Page

Experimental Details

S2

$\begin{array}{ll}\text { Figure S1. HPLC traces for BNPP and MNPP } & \text { S3 }\end{array}$

Figure S2. ESR spectra for BNPP and MNPP, experimental and simulated $\quad$ S4

Figure S3. UB3LYP/6-31G* DFT computed spin density distributions for BNPP $\quad$ S5

Figure S4. UB3LYP/6-31G* DFT computed spin density distributions for MNPP $\quad$ S6

Figure S5. $M$ vs $H$ plots for BNPP and MNPP $\quad$ S7

$\begin{array}{ll}\text { Figure S6. Curie plots for BNPP and MNPP } & \text { S7 }\end{array}$

Magnetic models used in fitting $\chi(T)$ data for BNPP and MNPP $\quad$ S8

Table S1. Selected bonding parameters and intermolecular distances. $\quad$ S9

$\begin{array}{ll}\text { Figure S5. Nitroxide-nitroxide contacts in MNPP } & \text { S10 }\end{array}$ 


\section{Experimental Details.}

General Methods. NMR analyses were carried out at facilities supported in part by National Science Foundation grant CHE-0234541. Magnetic analyses were carried out at facilities supported in part by National Science Foundation grant CTS-0116498. Crystallographic analyses were carried out at facilities supported in part by National Science Foundation grant CHE-9974648. Elemental analyses were carried out at the University of Massachusetts Amherst Microanalytical Laboratory. Electron spin resonance spectroscopy was carried out at facilities supported in part by National Science Foundation grant CHE-0443180.

ESR spectral studies. All ESR spectra were obtained in the X-band (9.6 GHz) using a T102 mode cavity. Samples of the appropriate radical (1-3 mg) were dissolved in about 500 microliters of toluene, placed in a stopcock-sealable $4 \mathrm{~mm} \mathrm{o.d}$. quartz tube, and subjected to freeze-pump-thaw degassing. Hyperfine analyses of the spectra were carried out using Duling's simulation program [Duling, D. R. J. Magn. Res. 1994, B104, 105].

Magnetic studies. Magnetic susceptibility $(\chi)$ versus temperature measurements were carried out over 1.8-300 K in a SQUID magnetometer, using polycrystalline samples (20-50 mg) placed in gelatin capsules and held in place with a small amount of cotton. Raw susceptibility data were corrected for temperature independent paramagnetic contributions $\left(\chi_{T I P}\right)$ based on the high temperature $\chi T(T)$ data. Magnetization versus field $\left(M_{\text {molar }}\right.$ vs $H$ ) measurements were carried out on the same samples at $1.8 \mathrm{~K}$ over $0-50 \mathrm{kOe}$.

4-Bromo- $N$-tert-butyl- $N$-(tert-butyldimethylsiloxy)aniline (BrPhNITBDMS). This compound was made by the method of Field \& Lahti (Field, L. M.; Lahti, P. M. Chem. Mater., 2003, $15,2861)$ to give materials with spectral characteristics as described in the literature report.

1-(4-(N-Tert-butyl- $N$-[tert-butyldimethylsiloxy]amino)phenyl)pyrrole (BNPPTBDMS). Into a round bottom flask were placed $\mathrm{NaO} t \mathrm{Bu}(0.44 \mathrm{~g}, 0.0046 \mathrm{~mol}), \mathrm{PdO}(\mathrm{Ac})_{2}(0.034 \mathrm{~g}, 0.155 \mathrm{mmol})$ and 1,1'-bis(diphenylphosphino)ferrocene (dppf, $0.102 \mathrm{~g}, 0.276 \mathrm{mmol}$ ). The flask was thoroughly flushed with nitrogen. BrPhNITBDMS (1.64 g, $0.0046 \mathrm{~mol})$ and pyrrole $(0.215 \mathrm{~g}, 0.0031 \mathrm{~mol})$ were placed in a second round bottom flask, flushed with nitrogen, dissolved in freshly distilled toluene, and transferred to the first flask via cannula. The mixture was then stirred and heated at $110^{\circ} \mathrm{C}$ for 2 days. Next, the reaction was allowed to cool, stirred with decolorizing carbon and filtered. The yellow colored solution was washed with water and extracted with toluene. The organic layer was separated and dried over anhydrous $\mathrm{K}_{2} \mathrm{CO}_{3}$, filtered, and rotary evaporated. The crude product was purified by flash chromatography (silica, 5: 95 Hexanes: dichloromethane, followed by 100\% dichloromethane) to give a yellow oil. ${ }^{1} \mathrm{H}$ NMR (acetone- $\left.d_{6}\right): \delta-0.01$ (broad s, $\left.6 \mathrm{H}\right), 0.92(\mathrm{~s}, 9 \mathrm{H}), 1.26(\mathrm{~s}, 9 \mathrm{H}), 6.25(\mathrm{t}$, $2 \mathrm{H}, \mathrm{J}=2.3 \mathrm{~Hz}$ ), $7.23(\mathrm{t}, 2 \mathrm{H}, \mathrm{J}=2.3 \mathrm{~Hz}$ ), 7.35 (broadened pseudo doublet, $2 \mathrm{H}, \mathrm{J} \sim 8 \mathrm{~Hz}$ ), 7.45 (AA'BB' m, $2 \mathrm{H}, \mathrm{J}=8.6 \mathrm{~Hz}$ ).

1-(4-(N-Tert-butyl- $N$-hydroxylamino)phenyl)pyrrole (BNPPH) from BNPPTBDMS. Conc $\mathrm{HCl}(0.5 \mathrm{~mL})$ was added to a solution of BNPPTMS $(0.100 \mathrm{~g}, 0.304 \mathrm{mmol})$ in $20 \mathrm{~mL}$ of $95 \%$ ethanol under nitrogen. The reaction mixture was stirred at room temperature overnight, rotovapped, diluted with water and neutralized with $1 \mathrm{M}$ aq $\mathrm{NaOH}$. The resulting aqueous solution was then extracted with dichloromethane. The organic layer was separated, dried over anhydrous $\mathrm{MgSO}_{4}$, filtered, and rotovapped. The resulting crude product was purified by column chromatography to give the product with mp 147-148 ${ }^{\circ} \mathrm{C}$. The product obtained from this route has essentially the same ${ }^{1} \mathrm{H}-\mathrm{NMR}$ and FTIR spectral properties as the product obtained by Route 2 given in the main text 
Figure S1. HPLC traces for BNPP (upper) and MNPP (lower) in methanol at $0.5 \mathrm{~mL}$ per minute, room temperature.

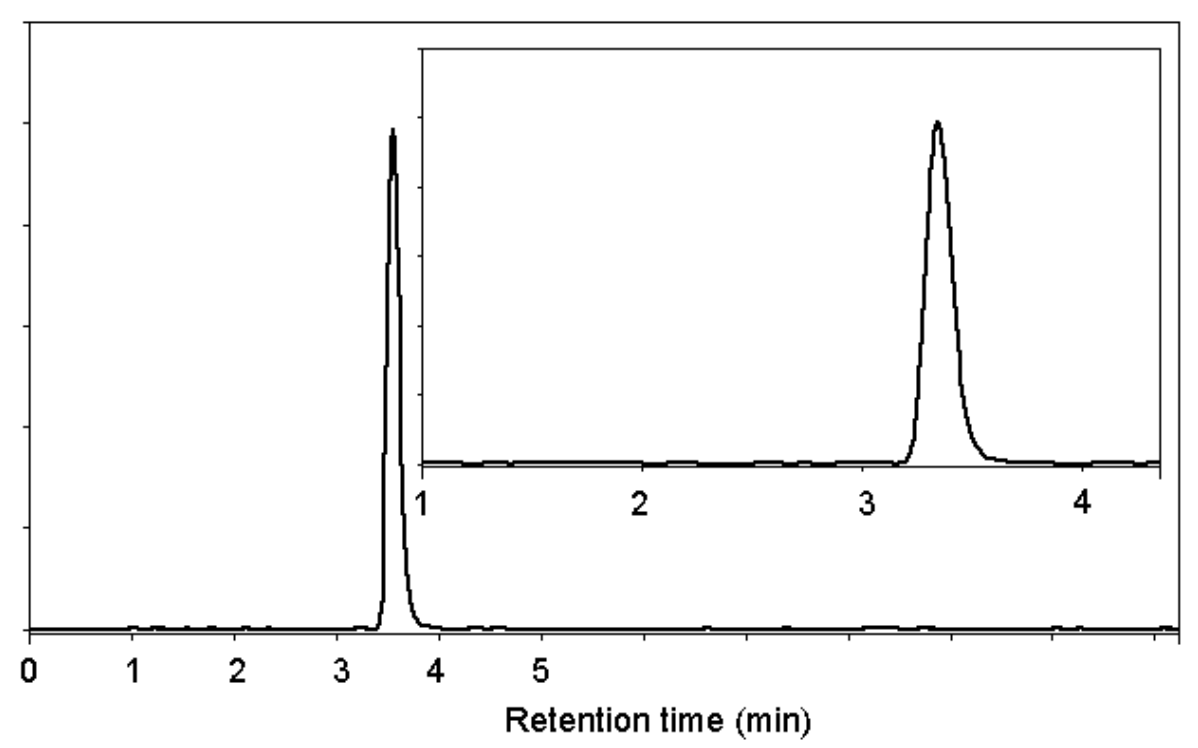<smiles>[CH2-]N(c1ccc(-n2cccc2)cc1)C(C)(C)C</smiles>
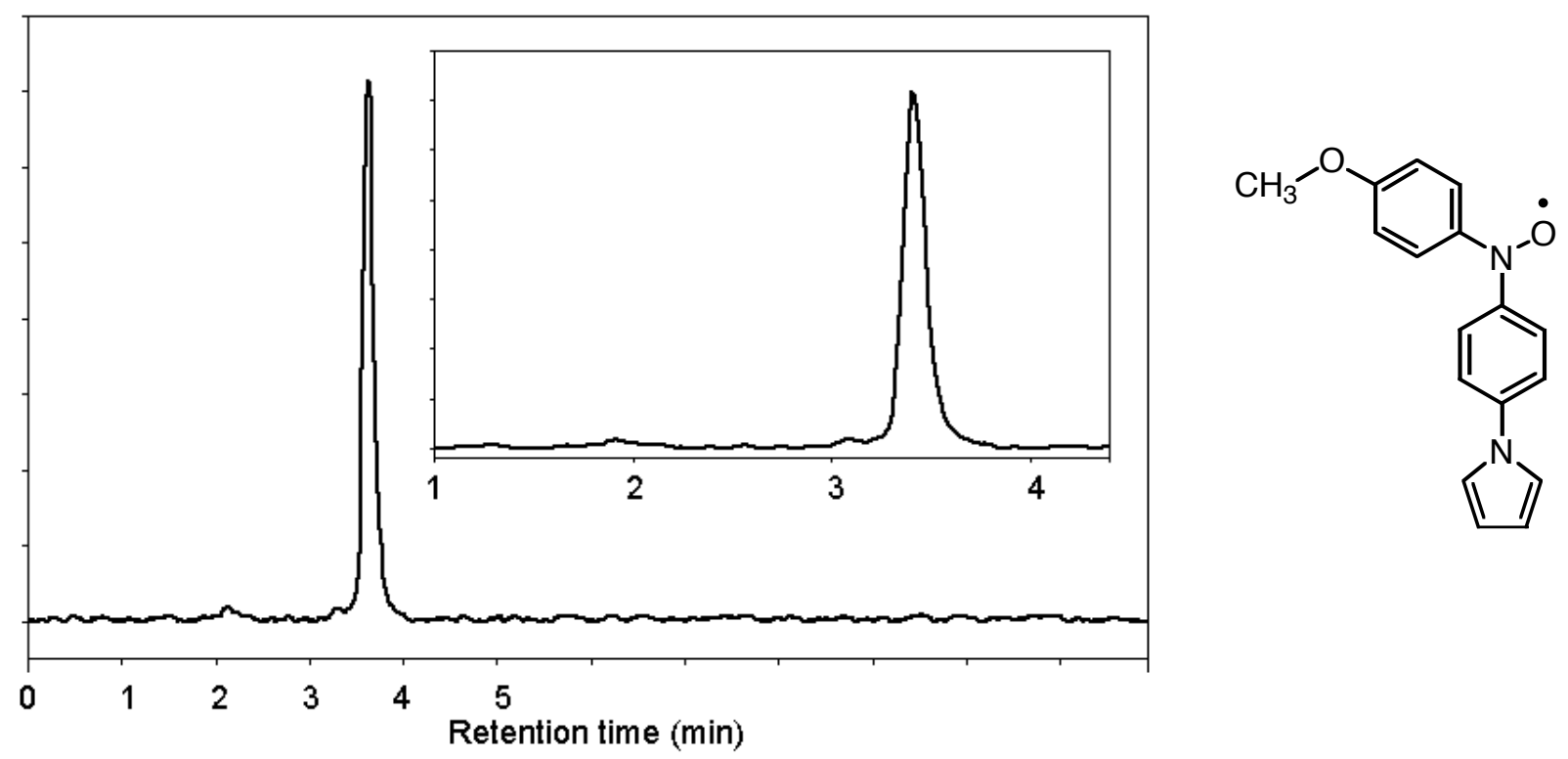
Figure S2. ESR spectra for BNPP and MNPP, experimental and simulated

Dat: 03/2006 Time: 16:58:42

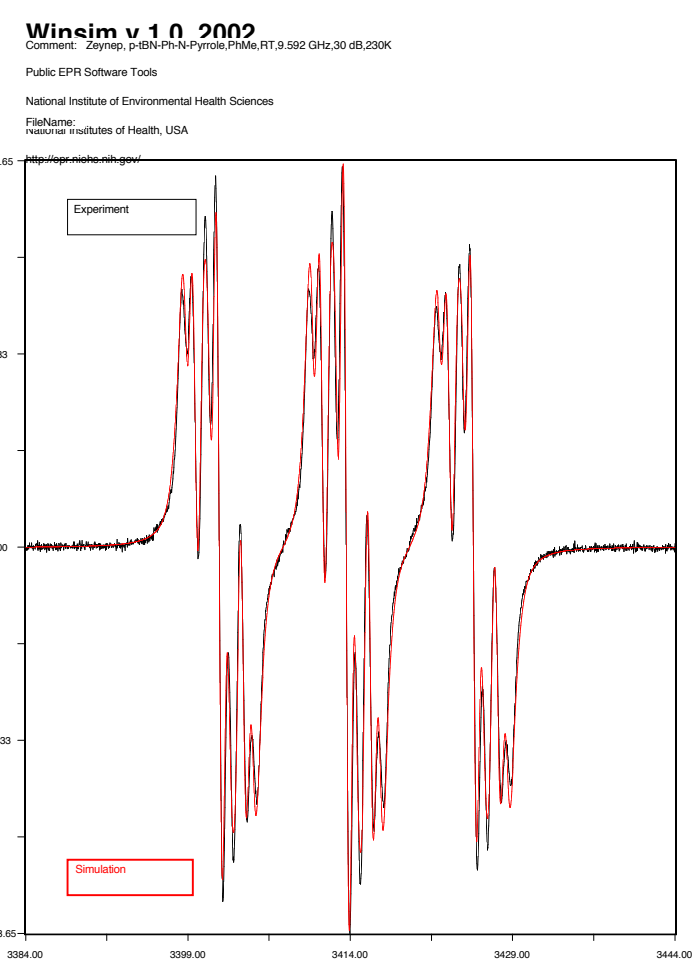

BNPP

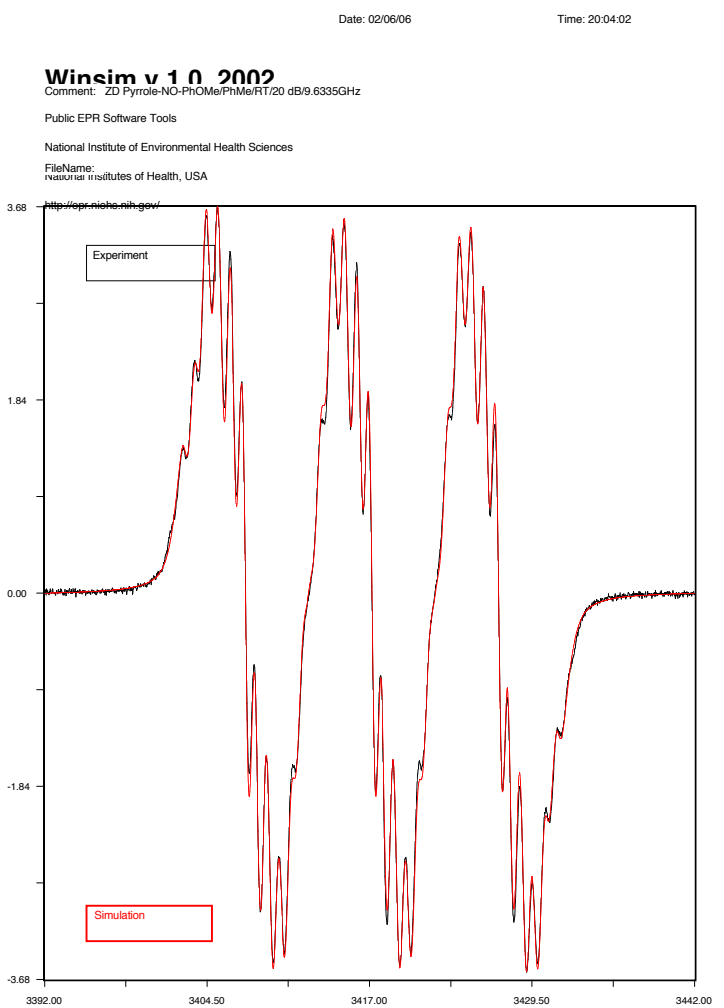

MNPP

Black lines are experimental spectral traces, red lines are simulated using the program WINSIM2002 downloaded from http://epr.niehs.nih.gov/pest.html in 2005 (see Duling, D. R. J. Magn. Res. 1994, B104, 105).

BNPP spectrum obtained in toluene at $230 \mathrm{~K}, 9.5923 \mathrm{GHz}, 30 \mathrm{~dB}$, time constant $2.56 \mathrm{~ms}$, modulation amplitude $0.029 \mathrm{G}$, modulation frequency $50 \mathrm{kHz}, 2048$ data points resolution for $60 \mathrm{G}$ sweep width. Simulated $\mathrm{a}_{\mathrm{N}}=11.74 \mathrm{G}, \mathrm{a}_{\mathrm{H} 1}=2.22 \mathrm{G}(2$ $\mathrm{H}), \mathrm{a}_{\mathrm{H} 2}=0.90 \mathrm{G}(2 \mathrm{H})$.

MNPP spectrum obtained in toluene at $296 \mathrm{~K}, 9.6332 \mathrm{GHz}, 20 \mathrm{~dB}$, time constant $10.24 \mathrm{~ms}$, modulation amplitude $0.101 \mathrm{G}$, modulation frequency $100 \mathrm{kHz}, 2048$ data points resolution for $50 \mathrm{G}$ sweep width. Simulated $\mathrm{a}_{\mathrm{N}}=9.73 \mathrm{G}, \mathrm{a}_{\mathrm{H} 1}=1.98 \mathrm{G}(2$ $\mathrm{H}), \mathrm{a}_{\mathrm{H} 2}=1.72 \mathrm{G}(2 \mathrm{H}), \mathrm{a}_{\mathrm{H} 3}=0.89 \mathrm{G}(2 \mathrm{H})$. 
Figure S3. UB3LYP/6-31G* DFT computed spin density distributions for BNPP.

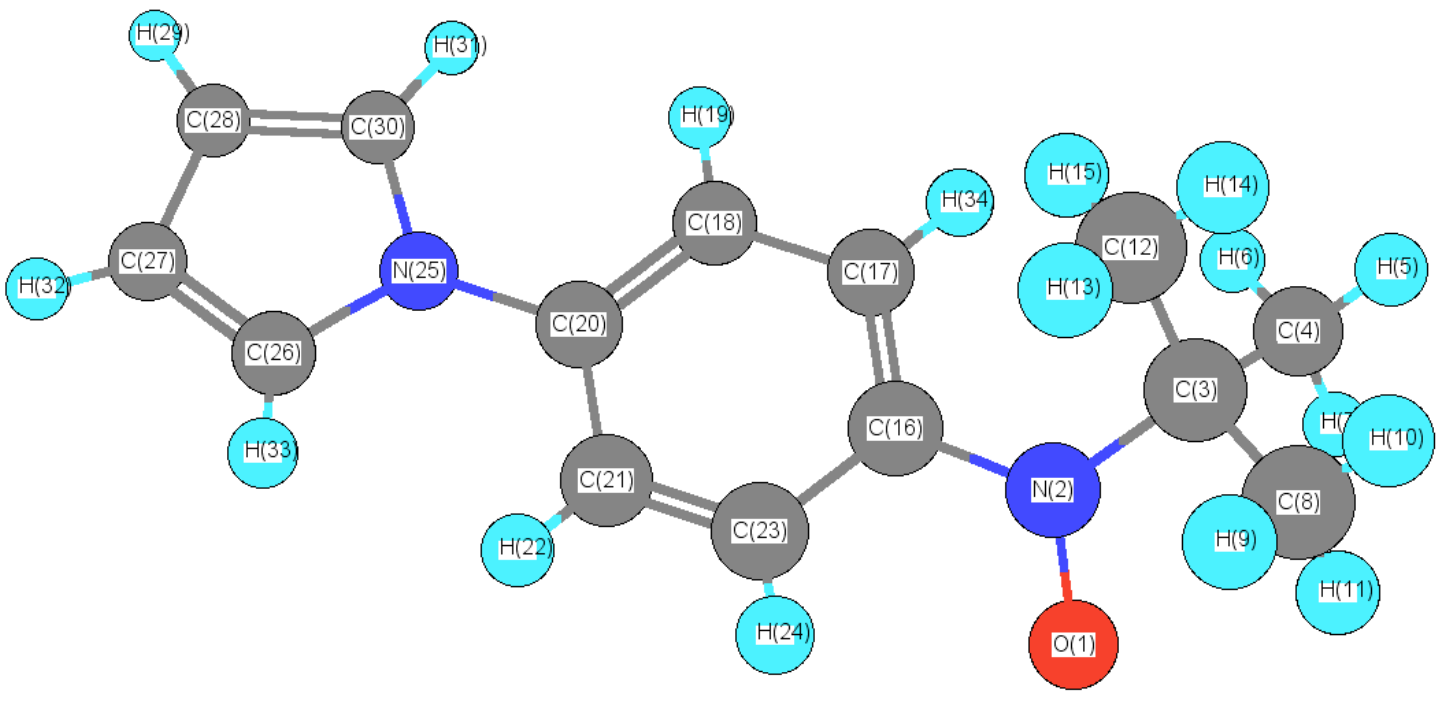

\begin{tabular}{|c|c|c|c|c|c|c|}
\hline$I$ & Atom & $\mathrm{X}$ & $\mathrm{Y}$ & $\mathrm{Z}$ & Charge (a.u.) & Spin $(a \cdot u \cdot)$ \\
\hline 列 & & & & & $0=1<0<0$ & C $50 \Omega$ \\
\hline 1 & $\mathrm{O}$ & 2.399903 & -1.847997 & -1.101720 & -0.416969 & 0.504604 \\
\hline 2 & $\mathrm{~N}$ & 2.074927 & -0.785015 & -0.481616 & -0.127178 & 0.393258 \\
\hline 3 & $\mathrm{C}$ & 3.179851 & 0.071378 & 0.060997 & 0.075184 & -0.025159 \\
\hline 4 & $\mathrm{C}$ & 3.550977 & 1.115542 & -0.972175 & -0.431137 & 0.020638 \\
\hline 5 & $\mathrm{H}$ & 4.280743 & 1.645306 & -0.643723 & 0.158312 & 0.002548 \\
\hline 6 & $\mathrm{H}$ & 2.796030 & 1.681844 & -1.140315 & 0.158430 & -0.000629 \\
\hline 7 & $\mathrm{H}$ & 3.811203 & 0.680272 & -1.787717 & 0.176339 & -0.001827 \\
\hline 8 & $\mathrm{C}$ & 4.365502 & -0.839798 & 0.332708 & -0.420967 & 0.004702 \\
\hline 9 & $\mathrm{H}$ & 4.108172 & -1.518836 & 0.960265 & 0.160982 & -0.000311 \\
\hline 10 & $\mathrm{H}$ & 5.088810 & -0.323865 & 0.699619 & 0.147779 & -0.001174 \\
\hline 11 & $\mathrm{H}$ & 4.647837 & -1.250384 & -0.487926 & 0.180186 & -0.000884 \\
\hline 12 & $\mathrm{C}$ & 2.753126 & 0.701093 & 1.374287 & -0.443119 & 0.006209 \\
\hline 13 & $\mathrm{H}$ & 2.502919 & 0.010894 & 1.993201 & 0.168670 & 0.000318 \\
\hline 14 & $\mathrm{H}$ & 3.482443 & 1.207963 & 1.737490 & 0.161417 & 0.000485 \\
\hline 15 & $\mathrm{H}$ & 2.005132 & 1.282200 & 1.221688 & 0.154063 & 0.000089 \\
\hline 16 & $\mathrm{C}$ & 0.673774 & -0.526832 & -0.357965 & 0.309760 & -0.081229 \\
\hline 17 & $\mathrm{C}$ & 0.122324 & 0.749800 & -0.318221 & -0.134041 & 0.091869 \\
\hline 18 & $\mathrm{C}$ & -1.241935 & 0.914696 & -0.212045 & -0.143673 & -0.047492 \\
\hline 19 & $\mathrm{H}$ & -1.596233 & 1.774814 & -0.182593 & 0.107351 & 0.001794 \\
\hline 20 & $\mathrm{C}$ & -2.095485 & -0.170076 & -0.147020 & 0.358349 & 0.083541 \\
\hline 21 & $\mathrm{C}$ & -1.534576 & -1.442161 & -0.210063 & -0.135954 & -0.047607 \\
\hline 22 & $\mathrm{H}$ & -2.089622 & -2.187523 & -0.179726 & 0.110747 & 0.001835 \\
\hline 23 & $\mathrm{C}$ & -0.183221 & -1.613332 & -0.315878 & -0.109006 & 0.086925 \\
\hline 24 & $\mathrm{H}$ & 0.167673 & -2.473922 & -0.358864 & 0.121654 & -0.004223 \\
\hline 25 & $\mathrm{~N}$ & -3.497508 & 0.002239 & -0.029018 & -0.558333 & -0.000383 \\
\hline 26 & $\mathrm{C}$ & -4.430520 & -0.983390 & 0.182197 & 0.054124 & 0.001953 \\
\hline 27 & $\mathrm{C}$ & -5.651114 & -0.404387 & 0.239262 & -0.150824 & 0.006576 \\
\hline 28 & $\mathrm{C}$ & -5.495688 & 0.955437 & 0.082508 & -0.142226 & 0.005916 \\
\hline 29 & $\mathrm{H}$ & -6.173887 & 1.590070 & 0.094532 & 0.092237 & -0.000315 \\
\hline 30 & $\mathrm{C}$ & -4.182593 & 1.195627 & -0.090847 & 0.051951 & 0.002730 \\
\hline 31 & $\mathrm{H}$ & -3.801270 & 2.033149 & -0.229955 & 0.130672 & -0.000161 \\
\hline 32 & $\mathrm{H}$ & -6.459104 & -0.848452 & 0.363259 & 0.092244 & -0.000349 \\
\hline 33 & $\mathrm{H}$ & -4.254408 & -1.892191 & 0.269967 & 0.133032 & -0.000127 \\
\hline 34 & $\mathrm{H}$ & 0.675818 & 1.495837 & -0.364594 & 0.109947 & -0.004121 \\
\hline Sum of & atomic & charges $=$ & 0.000000 & & & \\
\hline Sum of & spin & charges $=$ & 1.000000 & & & \\
\hline
\end{tabular}

Computed using UNIX Spartan 2000 (Wavefunction, Irvine, CA), UB3LYP/6-31G* at crystallographic geometry. 
Figure S4 UB3LYP/6-31G* DFT computed spin density distributions for MNPP.

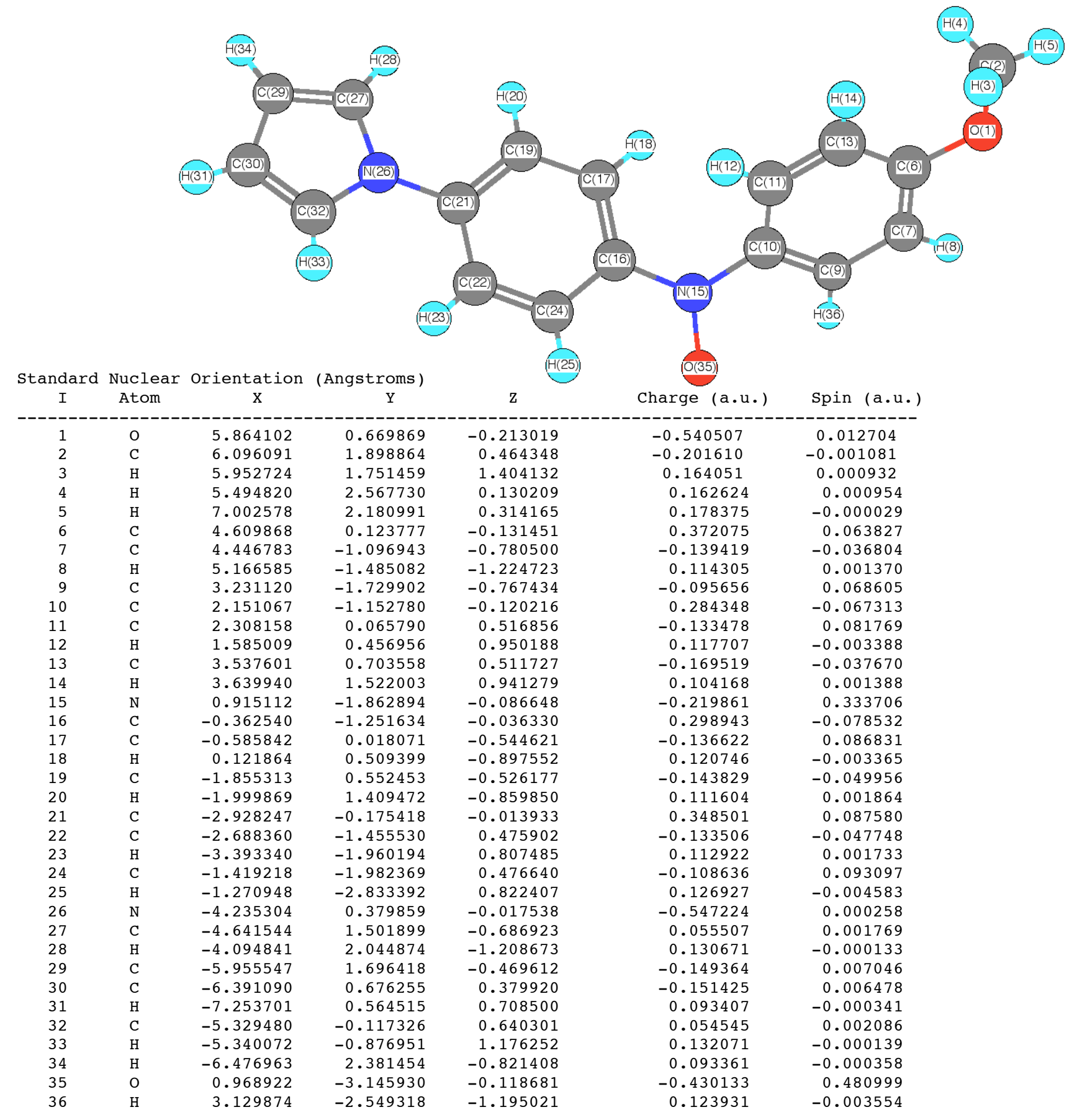

Mulliken Net Atomic Charges

$\begin{array}{ll}\text { Sum of atomic charges }= & 0.000000 \\ \text { Sum of spin charges }= & 1.000000\end{array}$

Computed using UNIX Spartan 2000 (Wavefunction, Irvine, CA), UB3LYP/6-31G* at crystallographic geometry. 
Figure S5. Magnetization $(M)$ versus field (H) for BNPP (circles) and MNPP (triangles) at $1.8 \mathrm{~K}$.

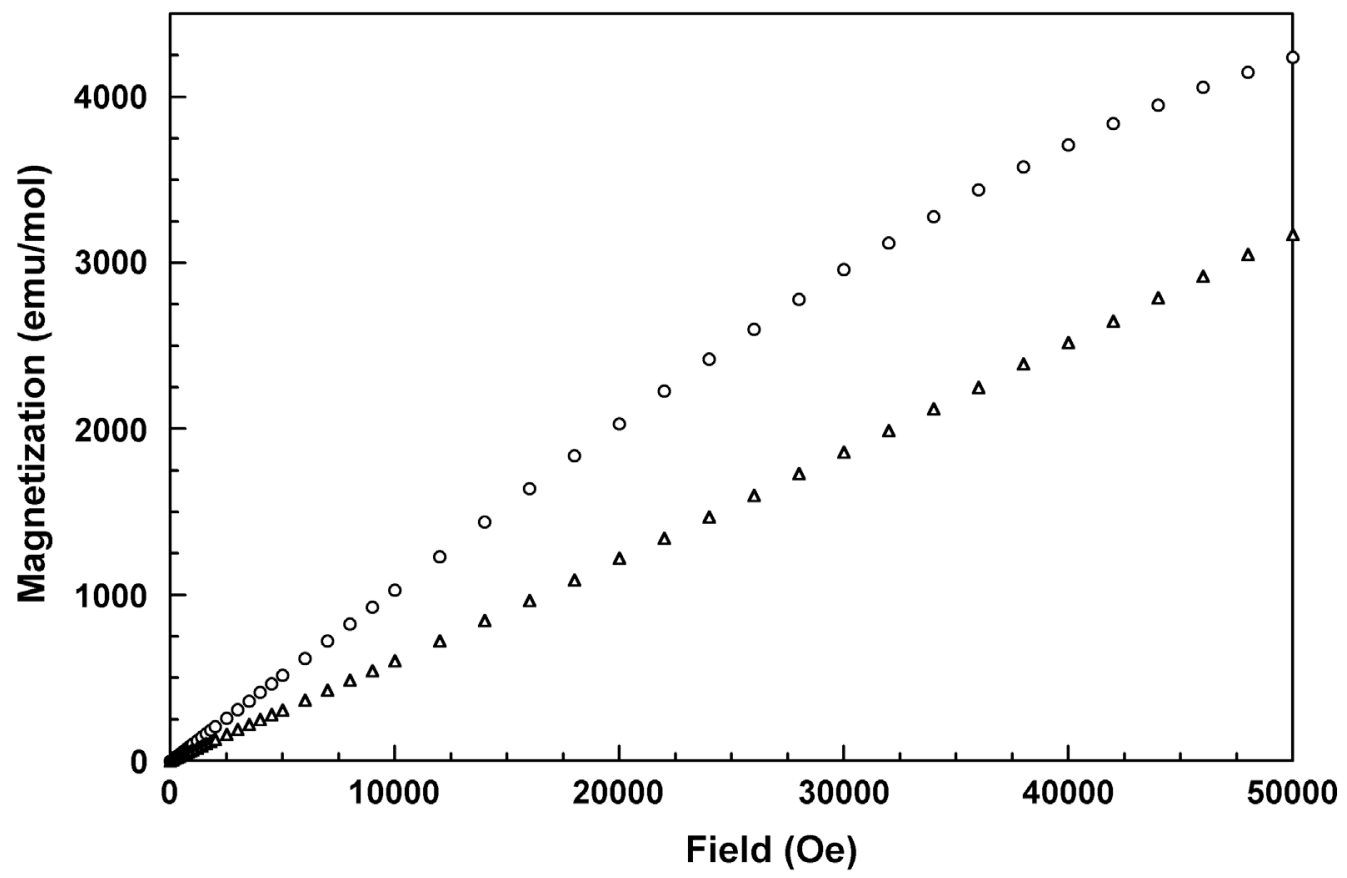

The limiting (saturation) value for a pure sample of $S=1 / 2$ systems at high fields is expected to be about $5585 \mathrm{emu} / \mathrm{mol}$. The curves above show the presence of antiferromagnetic exchange interactions, more strongly for MNPP than for BNPP.

Figure S6. Curie plots for MNPP (left) and BNPP (right) at 1000 Oe.
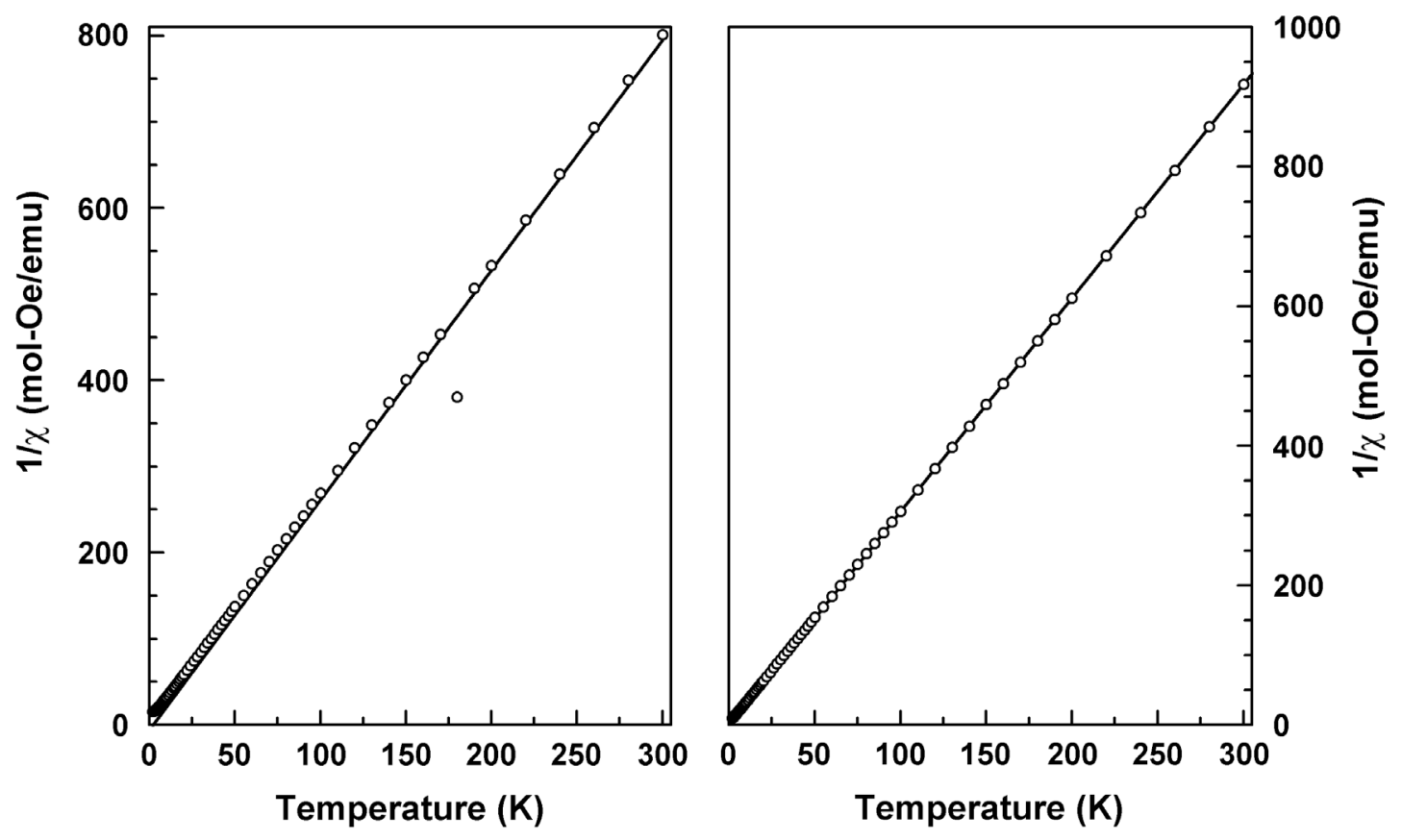

Slope $=2.66839464 \pm 0.108732925($ std dev $)$ for $\mathrm{T}>100 \mathrm{~K}$

Slope $=3.06056491 \pm 0.0020401791($ std dev $)$ For $\mathrm{T}>100 \mathrm{~K}$ 


\section{Magnetic models used in fitting $\chi(T)$ data for BNPP and MNPP}

Equations and constants used for fitting $\chi$ vs $\mathrm{T}$ data in main paper:
Bohr Magneton
$\beta=9.274 \times 10^{-24} \mathrm{~J} \mathrm{~T}^{-1}$
Boltzmann constant
$\mathrm{k}=1.3807 \times 10^{-23} \mathrm{~J} \mathrm{~K}^{-1}$
Avogadro's constant
$\mathrm{N}=6.0220 \times 10^{23} \mathrm{~mol}^{-1}$

$\mathrm{N} \beta^{2} / \mathrm{k}=0.375 \mathrm{emu} \mathrm{K} / \mathrm{mol}$

$\underline{\text { Spin-Pairing of two } \mathrm{S}=1 / 2 \text { systems (Bleaney-Bowers model) }}$

Bleaney, B.; Bowers, K. D. Proc. R. Soc. London, A, 1952, 214. (S-T gap is 2J, not J)

$\chi=\frac{2 N g^{2} \beta^{2}}{3 k T} \frac{3}{3+\exp (-2 J / k T)}=0.375 \cdot\left(\frac{g^{2}}{T}\right) \cdot \frac{2}{3+\exp (-2 J / k T)}$

Hamiltonian used is

$\hat{H}_{12}=2 J_{12} \hat{S}_{1} \hat{S}_{2}$

AFM Bonner-Fisher linear chain, $\mathrm{S}=1 / 2$ units, all $\mathrm{J}$ equal

(a) Bonner, J. C.; Fisher, M. E. Phys. Rev. A, 1964, 135, 650. J. C. (b) Bonner, Ph. D. Dissertation, University of London, UK, 1968.

(b) $=\frac{N g^{2} \beta^{2}}{k(T-\theta)} \cdot \frac{A+B \cdot(J / k T)+C \cdot(J / k T)^{2}}{1+D \cdot(J / k T)+E \cdot(J / k T)^{2} F \cdot(J / k T)^{3}}$

$\mathrm{A}=0.25, \mathrm{~B}=0.14995, \mathrm{C}=0.30094, \mathrm{D}=1.9862, \mathrm{E}=0.68854$ and $\mathrm{F}=6.0626$

Hamiltonian used is

$\hat{H}_{12}=J_{12} \hat{S}_{1} \hat{S}_{2}$

$\underline{\text { Square-planar antiferromagnetic sheet, all } \mathrm{S}=1 / 2 \text { units }}$

Baker, G., Jr.; Gilbert, H. E.; Eve, J.; Rushbrooke, G. S. Phys Lett, 1967, 25A(3), 207.

$\chi=\frac{N g^{2} \beta^{2}}{k(T-\theta)} \cdot\left[1+\frac{4 x}{2}+\frac{16 x^{2}}{(2 !)\left(2^{2}\right)}+\frac{64 x^{3}}{(3 !)\left(2^{3}\right)}+\frac{416 x^{4}}{(4 !)\left(2^{4}\right)}+\frac{4544 x^{5}}{(5 !)\left(2^{5}\right)}+\frac{23488 x^{6}}{(6 !)\left(2^{6}\right)}-\frac{207616 x^{7}}{(7 !)\left(2^{7}\right)}+\frac{4205056 x^{8}}{(8 !)\left(2^{8}\right)}+\frac{198295552 x^{9}}{(9 !)\left(2^{9}\right)}-\frac{2574439424 x^{10}}{(10 !)\left(2^{10}\right)}\right]$ $x=J / k T$

Hamiltonian used is

$\hat{H}_{12}=J_{12} \hat{S}_{1} \hat{S}_{2}$ 


\begin{tabular}{|c|c|c|c|}
\hline BNPP & & MNPP & \\
\hline Parameter & Value & Parameter & Value \\
\hline $\mathrm{N}(1) \mathrm{O}(1)$ & $1.273(3)$ & $\mathrm{N}(1) \mathrm{O}(2)$ & $1.284(5)$ \\
\hline$N(1) C(5)$ & $1.431(3)$ & $N(1) C(8)$ & $1.417(6)$ \\
\hline$N(2) C(10)$ & $1.416(3)$ & $N(2) C(11)$ & $1.420(7)$ \\
\hline$C(5) C(7)$ & $1.391(3)$ & $C(8) C(9)$ & $1.383(7)$ \\
\hline $\mathrm{C}(7) \mathrm{C}(9)$ & $1.366(3)$ & $\mathrm{C}(9) \mathrm{C}(10)$ & $1.374(4)$ \\
\hline $\mathrm{O}(1) \mathrm{N}(1) \mathrm{C}(1) \mathrm{C}(2)$ & $27.3(2)$ & $\mathrm{O}(2) \mathrm{N}(1) \mathrm{C}(8) \mathrm{C}(9)$ & $24.3(5)$ \\
\hline $\mathrm{C}(9) \mathrm{C}(10) \mathrm{N}(2) \mathrm{C}(12)$ & $7.6(2)$ & $\mathrm{C}(10) \mathrm{C}(11) \mathrm{N}(2) \mathrm{C}(14)$ & $12.4(6)$ \\
\hline \multirow{2}{*}{$\mathrm{C}(7) \mathrm{C}(5) \mathrm{N}(1) \mathrm{O}(1)$} & $29.6(2)$ & $\mathrm{O}(2) \mathrm{N}(1) \mathrm{C}(5) \mathrm{C}(4)$ & $29.0(5)$ \\
\hline & & $\mathrm{C}(1) \mathrm{O}(1) \mathrm{C}(2) \mathrm{C}(7)$ & $2.9(6)$ \\
\hline$O(1) \cdots C\left(3^{\prime}\right)$ & $4.012(4)$ & $\mathrm{O}(2) \cdots \mathrm{N}\left(1^{\prime}\right)$ & $4.062(7)$ \\
\hline$O(1) \cdots C\left(2{ }^{\prime \prime}\right)$ & $3.517(4)$ & $O\left(2^{\prime}\right) \cdots N\left(1^{\prime \prime}\right)$ & $4.148(7)$ \\
\hline$O\left(1^{\prime}\right) \cdots C\left(3^{\prime \prime}\right)$ & $3.647(4)$ & $O\left(2^{\prime \prime}\right) \cdots N\left(1^{\prime \prime \prime}\right)$ & $4.062(7)$ \\
\hline$O\left(1^{\prime \prime \prime \prime}\right) \cdots C\left(13^{\prime}\right)$ & $3.759(4)$ & $O(2) \cdots O\left(2^{\prime}\right)$ intrachain & $4.778(7)$ \\
\hline$N(1) \cdots C\left(3^{\prime}\right)$ & $3.857(3)$ & $O\left(2^{\prime}\right) \cdots O\left(2^{\prime \prime}\right)$ intrachain & $4.848(7)$ \\
\hline$C\left(7^{\prime}\right) \cdots C(11)$ & $3.693(4)$ & & \\
\hline$C\left(12^{\prime}\right) \cdots C(6)$ & $3.980(4)$ & $\mathrm{O}(2) \cdots \mathrm{O}\left(2^{\prime}\right)$ interchain & $4.778(7), 4.848(7)$ \\
\hline$C\left(14^{\prime}\right) \cdots C(6)$ & $4.073(4)$ & & \\
\hline$O\left(1^{\prime}\right) \cdots O\left(1^{\prime} b\right)$ & $5.8054(2)$ & & \\
\hline \multirow[t]{2}{*}{$\mathrm{O}\left(1^{\prime}\right)^{\prime} \cdots \mathrm{N}\left(1^{\prime} \mathrm{b}\right)$} & $5.697(3)$ & & \\
\hline & & $\mathrm{N}(1) \mathrm{O}(2) \cdots \mathrm{N}\left(1^{\prime}\right)$ & 116.5 \\
\hline $\mathrm{N}\left(1^{\prime}\right) \mathrm{O}\left(1^{\prime}\right) \cdots \mathrm{N}\left(1^{\prime} b\right)$ & 88.5 & $\mathrm{~N}\left(1^{\prime}\right) \mathrm{O}\left(2^{\prime}\right) \cdots \mathrm{N}\left(1^{\prime \prime}\right)$ & 116.1 \\
\hline
\end{tabular}


Figure S7. Nitroxide-nitroxide contacts in MNPP. Distances in angstroms.

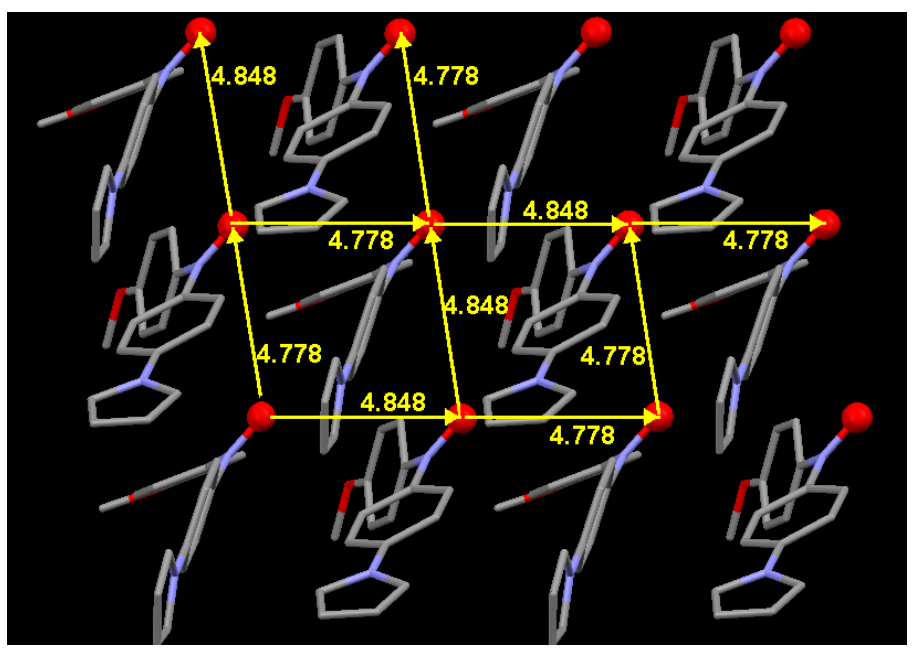

